ACCEPTED MANUSCRIPT

\title{
The role of hairs in the adhesion of octopus suckers: a hierarchical peeling approach
}

To cite this article before publication: Gabriele Greco et al 2020 Bioinspir. Biomim. in press https://doi.org/10.1088/1748-3190/ab72da

\section{Manuscript version: Accepted Manuscript}

Accepted Manuscript is "the version of the article accepted for publication including all changes made as a result of the peer review process, and which may also include the addition to the article by IOP Publishing of a header, an article ID, a cover sheet and/or an 'Accepted Manuscript' watermark, but excluding any other editing, typesetting or other changes made by IOP Publishing and/or its licensors"

This Accepted Manuscript is @ 2020 IOP Publishing Ltd.

During the embargo period (the 12 month period from the publication of the Version of Record of this article), the Accepted Manuscript is fully protected by copyright and cannot be reused or reposted elsewhere.

As the Version of Record of this article is going to be / has been published on a subscription basis, this Accepted Manuscript is available for reuse under a CC BY-NC-ND 3.0 licence after the 12 month embargo period.

After the embargo period, everyone is permitted to use copy and redistribute this article for non-commercial purposes only, provided that they adhere to all the terms of the licence https://creativecommons.org/licences/by-nc-nd/3.0

Although reasonable endeavours have been taken to obtain all necessary permissions from third parties to include their copyrighted content within this article, their full citation and copyright line may not be present in this Accepted Manuscript version. Before using any content from this article, please refer to the Version of Record on IOPscience once published for full citation and copyright details, as permissions will likely be required. All third party content is fully copyright protected, unless specifically stated otherwise in the figure caption in the Version of Record.

View the article online for updates and enhancements. 


\title{
The role of hairs in the adhesion of octopus suckers: a hierarchical peeling approach
}

\author{
Gabriele Greco ${ }^{1,2}$; Federico Bosia ${ }^{3}$, Francesca Tramacere ${ }^{2}$; Barbara Mazzolai² and Nicola M. \\ Pugno ${ }^{1,4,5^{*}}$
}

${ }^{1}$ Laboratory of Bio-Inspired \& Graphene Nanomechanics, Department of Civil, Environmental and Mechanical Engineering, University of Trento, Via Mesiano, 77, 38123 Trento, Italy

${ }^{2}$ Center for Micro-BioRobotics@SSSA, Istituto Italiano di Tecnologia, Viale Rinaldo Piaggio 34, 156025 Pontedera, Italy

${ }^{3}$ Department of Applied Science and Technology, Politecnico di Torino, Corso Duca degli Abruzzi 24, 10129, Torino, Italy

${ }^{4}$ School of Engineering and Materials Science, Queen Mary University of London, Mile End Road, E1 4NS London, United Kingdom

${ }^{5}$ Ket-Lab, Edoardo Amaldi Foundation, Via del Politecnico snc, 00133 Rome, Italy

*Corresponding author: nicola.pugno@unitn.it

\begin{abstract}
Organisms like the octopus or the clingfish are a precious source of inspiration for the design of innovative adhesive systems based on suction cups, but a complete mechanical description of their attachment process is still lacking. In this paper, we exploit the recent discovery of the presence of hairs in the acetabulum roof of octopus suction cups to revise the current model for its adhesion to the acetabulum wall. We show how this additional feature, which can be considered an example of a hierarchical structure, can lead to an increase of adhesive strength, based on the analysis of the cases of a simple tape and an axisymmetrical membrane. Using peeling theory, we discuss in both cases the influence of hierarchical structure and the resulting variation of contact angles on the adhesive energy, highlight how an increase in number of hierarchical levels contributes to its increase, with a corresponding improvement in functionality for the octopus suckers.
\end{abstract}

\section{Introduction}

The Octopus vulgaris is one of the most intelligent animals that lives on Earth. It uses its suckers to perform many functions ([1], [2]). In particular, octopus suckers are able to generate a maximum pressure difference of about 0.27 MPa that can be reached in a few milliseconds [3]. Other animals, such as clingfish, exploit suction cups with a bed of microfibrils or "micropapillae", which are tiny soft protuberances that line the cup perimeter, to better adhere to rough rock surfaces underwater [4]. For this reason, these structures represent a remarkable source of inspiration for designing 34 artificial suction cups or adhesives ([5]-[8]). To develop these artificial devices, the full 
understanding of the adhesion process and the capability to model it correctly is crucial. In the past, octopus suckers and their interaction with the substrate have been studied mainly by analyzing their arrangement [9] and structure ([10], [11]). In Tramacere et al.[9], a method to identify the suckers in the octopus arm was developed in order to better determine its mechanics through imaging. Moreover, in Tramacere et al. [10], three techniques (MRI, ultrasonography, and histology) were used to gain a 3D reconstruction of the sucker (Fig. 1). In this context, the acetabulum protuberance in the acetabulum cavity was discovered for the first time. Experimental studies were also performed to measure the full mechanical properties of the octopus sucker tissues in [11]. Unfortunately, a reliable value of the Poisson ratio remains to be obtained. Work is in progress to resolve this issue. The adhesion of the octopus suckers is achieved by exploiting the pressure difference between the external environment, the acetabulum cavity and the infundimbulum cavity (Fig. 1a) [12]. To maintain this pressure difference, the acetabulum roof and the acetabulum wall must remain in full contact [10]. More in detail, at the initial stage of adhesion, the infundimbulum is the first part of the sucker in contact with the substrate to form a seal. Then, the acetabular radial muscles contract to reduce the internal pressure in the sucker with respect to the external one. Finally, the meridional muscle of the acetabulum contracts to achieve contact between the acetabulum roof and the acetabulum cavity. At this point, all muscles are contracted. When they relax, the adhesion is maintained by the adhesive force maintaining the two surfaces in contact (the acetabulum roof and the acetabulum cavity) [13]. Morphological studies show that the latter does not present any hairs and can be considered flat.

As in other bioadhesion problems, peeling theory has been adopted to describe how these two parts of the octopus suckers delaminate [14]. The first elastic approach developed in the literature in this respect was the Kendall model [15], which describes the peeling of a thin elastic tape from a rigid substrate. The main physical quantity that governs the attachment, or the detachment, of the tape is the surface energy $\gamma$, which is defined as the energy required to generate a unit area of interface (for a certain crack speed), with Mode I (opening) primary separation mode. In the Kendall model, the force necessary to detach the membrane can be determined by adopting an energy-based criterion, imposing the Griffith's balance between the elastic energy, the adhesive energy and the 63 work of the applied load [16]. The peeling force relative to a tape pulled at an angle $\theta$, is thus:

$$
F=E t w\left(\cos \alpha_{0}-1+\sqrt{\left(1-\cos \alpha_{0}\right)^{2}+\frac{2 \gamma}{E t}}\right)
$$


65 where $E$ is the Young's modulus of the tape, $t$ its thickness and $w$ its width. Introducing $\hat{F}=$ $F /(E t w)$, where Etw represents the force necessary to generate a unit strain in the tape, and $\hat{\gamma}=$ $\gamma /(E t)$, the relation can be written in non-dimensional form:

$$
\hat{F}=\cos \alpha_{0}-1+\sqrt{\left(1-\cos \alpha_{0}\right)^{2}+2 \hat{\gamma}}
$$

Starting from this approach, a series of more refined models were developed in order to describe various biological mechanisms of adhesion. Among these, the theory of multiple peeling was introduced to model a system of numerous tapes loaded by a single force at a common point [17]. This was used in complex adhesive systems, e.g. to describe the adhesive behaviour of spider web anchors [18],[19],[20]. Effects such as tape geometry, viscoelasticity or surface roughness [21], [22] have also been considered, as well as bending stiffness[23]. Moreover, a so-called "hierarchical shear lag model" was introduced to model hierarchical contact splitting occurring in biological adhesive structures such as gecko pads [24], [25], which are suitable for active dynamic short-term attachment, and other approaches have considered the effect of pretension in hierarchical structures [26]. These works showed that hierarchical structuring of the surface also leads to the reduction of stress concentrations and the appearance of multiple separate peeling fronts, with a resulting increase in adhesive capabilities. These examples indicate the possibility of exploiting various types of structures present in nature for enhanced adhesion in artificial adhesives.

The recent discovery of the presence of hairs in the acetabulum roof of the octopus' suckers [27] (Fig. 1) suggests a revision of the model outlined in Tramacere et al. [13]. In particular, the peeling model therein can be improved by adding the additional effect due to the presence of hairs on the flat membrane. This work therefore aims to model the peeling process of a membrane equipped with hierarchical hairs, i.e. to analyse how the hairs affect the peeling force. To do this, we apply Yao's approach [28] to the geometry of an axisymmetric membrane, formulating a modified expression for the work of adhesion as a function of the surface energy in a hierarchical structure and deriving the corresponding detachment force of the membrane.

\section{Theoretical model}

\subsection{Hierarchical tape with hairs}

We analyse a simple tape with hairs at the interface with the substrate, as shown schematically in 
present at two (or more) different size scales. As a first approximation, hairs are considered to be of the same material of the tape (an incompressible soft material with $v=0.5$ ). Furthermore, they are modelled as flat tapes of thickness $t_{1}$, width $w_{1}$ detached length $L_{1}$ and contact length $I_{1}$. The distance between two adjacent hairs is $\rho$ along both $x$ and $y$ directions, so that $N=l w / \rho^{2}$ is the total number of hairs. The hairs form an angle $\alpha_{1}$ with the substrate that is considered to be constant, and whose relation to the tape contact angle $\alpha_{0}$ is discussed below. During the attachment and detachment phases, we do not consider bunching effects of the hairs and possible variation effects in the section of the tape. Equation (1) is valid for a simple tape without hairs. The presence of hairs on the tape surface results in an increase of the equivalent surface energy, since there is additional elastic energy stored in the hairs themselves that is "dissipated" as kinetic energy released after detachment ([25], [29]). Thus, Eq. (1) remains valid and the surface energy term can be modified to

$$
\gamma^{\prime}=\gamma+\gamma_{H}
$$

where $\gamma^{\prime}$ is the total surface energy, $\gamma$ the surface energy of the flat tape and $\gamma_{H}$ the equivalent surface energy due to the additional elastic energy stored in the hairs. As a first approximation, we neglected the roughness of the substrate. According to previous work [22], this roughness is not expected to influence results significantly, unless it is of the order of the microscopic features (i.e. the hairs) of the adhesive surface, which is not the case considered herein.

Since all hairs are assumed identical, $\gamma_{H}$ can be considered homogeneous over the whole contact surface, and can be evaluated as:

$$
\gamma_{H}=\frac{l_{1}+L_{1}}{2 E w_{1}^{2} t_{1} l_{1}} \quad P_{1}^{2}
$$

where $P_{1}$ is the detachment force of a single hair. Using Eq. (1) to compute $P_{1}$, we obtain:

$$
\gamma_{H}=\frac{E t_{1}}{2}\left(1+\frac{L_{1}}{l_{1}}\right)\left(\cos \alpha_{1}-1+\sqrt{\left(1-\cos \alpha_{1}\right)^{2}+\frac{2 \gamma}{E t_{1}}}\right)^{2}
$$

We can now write Eq. (3) in non-dimensional form:

$$
\widehat{\gamma^{\prime}}=\hat{\gamma}+\frac{\gamma_{H}}{E t}=\hat{\gamma}+\frac{t_{1}}{2 t}\left(1+\frac{L_{1}}{l_{1}}\right)\left(\cos \alpha_{1}-1+\sqrt{\left(1-\cos \alpha_{1}\right)^{2}+\frac{2 t}{t_{1}}} \hat{\gamma}\right)^{2}
$$

Substituting this expression for the surface energy in Eq. (2), we obtain the modified nondimensional pull-off force as: 


$$
\hat{F}=\cos \alpha_{0}-1+\sqrt{\left(1-\cos \alpha_{0}\right)^{2}+2 \hat{\gamma}+\kappa_{1}\left(\cos \alpha_{1}-1+\sqrt{\left(1-\cos \alpha_{1}\right)^{2}+\lambda_{1} \hat{\gamma}}\right)^{2}}
$$

123 Where $\kappa_{1}=\frac{t_{1}}{2 t}\left(1+\frac{L_{1}}{l_{1}}\right)$ and $\lambda_{1}=\frac{2 t}{t_{1}}$. Equation (7) thus represents the dimensionless force necessary to detach a rectangular tape equipped with hairs. Notice that the area fraction, i.e. the ratio between the contact areas of the tape with/without hairs, respectively, is usually considered close to 1, i.e., the presence of hairs does not entail a reduction/increase of the contact area[25]. To illustrate the resulting behavior, we plot the peeling force $\hat{F}$ in Fig. $3 \mathrm{~b}$ for various angles $\epsilon$, having chosen the following parameters: $\hat{\gamma}=4 \cdot 10^{-4}, w=10^{-2} \mathrm{~m}, l=10^{-2} \mathrm{~m}, t=10^{-3} \mathrm{~m}, w_{1}=10^{-5} \mathrm{~m}, l_{1}=$ $10^{-5} \mathrm{~m}, L_{1}=10^{-5} \mathrm{~m}, t_{1}=10^{-5} \mathrm{~m}$. As expected, the presence of a hierarchical structure, i.e. of hairs, contributes to an increase of the adhesive properties of the tape for all peeling angles due to the additional stored elastic energy, which is dissipated during delamination, with an increased effect for small angles. The peeling force decreases only slightly for increasing $\epsilon$ values. For $\alpha_{0}=0$, and $\alpha_{1}=0$, the tape is sheared parallel to the surface, and the additional dissipated energy due to the contribution of the hairs is maximum. Conversely, their decreasing effect when the peeling angle increases and tends to $\pi / 2$ is consistent with the qualitative behavior observed in biological adhesion, where the peeling force needs to be maximized mainly for small peeling angles, while facilitated detachment is required at larger angles, to achieve the ON/OFF mechanism necessary, e.g. for motion in animals like geckos or insects like beetles.

It should be noted that in Eq.(1) and its derivations, we neglect the effect of the deformation of the substrate. In previous work, the presence of a soft substrate in peeling problems was seen to give rise to an overall increase in the detachment force, due to a wider load distribution at the interface, reducing the load concentration at the peeling line, and a decrease of the local peeling angle [22]. Thus, we expect the soft substrate not to affect the predicted qualitative behavior.

\subsection{Hierarchical axisymmetric membrane}

The detachment of a single octopus' sucker can be treated as the peeling of an axisymmetric membrane [13], treated by Afferrante et al. [30], and schematically illustrated in Fig. 4a. The nondimensional force necessary to detach the membrane is

$$
\widehat{F}=\left(\frac{32}{27}\right)^{\frac{1}{4}}(\hat{\gamma})^{\frac{3}{4}}(1+\hat{a})
$$


150 where $\hat{F}$ and $\hat{a}$ are the dimensionless normal load and detached radius, respectively. Equation (8) 151 predicts a linearly increasing peeling force with the membrane detached radius $\hat{a}$, i.e. an adhesive 152 membrane can ideally bear an arbitrary load, provided it is large enough. In this case, the 153 modification of $y$ due to the presence of hairs should be also considered. By inserting Eq. (6) in Eq. 10 with hairs, although in this case the latter are assumed to be radially distributed, as shown in Fig. 4b. Making the same assumptions as in the previous Section, we obtain the detachment force of the axisymmetric membrane as:

$$
\hat{F}=\left(\frac{32}{27}\right)^{\frac{1}{4}}\left(\hat{\gamma}+\kappa_{1}\left(\cos \alpha_{1}-1+\sqrt{\left(1-\cos \alpha_{1}\right)^{2}+\lambda_{1} \hat{\gamma}}\right)^{2}\right)^{3 / 4}(1+\hat{a})
$$

The role of the hairs for the axisymmetric membrane can be visualized in Fig. 5 . In this case, we plot the peeling force versus the detached radius $\hat{a}$ for $\hat{\gamma}=4 \cdot 10^{-4}$, and various values of $\alpha_{1}$. The dependence is linear, but again, the presence of a hierarchical structure implies a considerable increase in the adhesive properties of the membrane for a given detached radius. The influence of the hairs on the peeling force decreases as the angle increases, but the $\hat{F}$ vs. $\hat{a}$ curves remain considerably larger than that relative to non-hierarchical case, even for large angles, e.g. $\alpha_{1}=0.4$. This is again consistent with the qualitative behavior observed in biological adhesion, where the peeling force needs to be maximized mainly for small peeling angles.

It should be noted that in Eq.(1) and its derivations, we neglect the effect of the deformation of the substrate. In previous work [22], the presence of a soft substrate in peeling problems was seen to give rise to an overall increase in the detachment force, due to a wider load distribution at the interface, reducing the load concentration at the peeling line, and a decrease of the local peeling angle. Thus, we expect the soft substrate not to affect the predicted qualitative behaviour.

\subsection{Additional levels of hierarchy}

The previous model can be extended to additional levels of hierarchy, as illustrated schematically in Fig. 3a. In this case, Eq. (3) can be extended as follows:

$$
\gamma^{\prime}=\gamma+\gamma_{1}+\gamma_{2}+\cdots+\gamma_{n}
$$

where $\gamma_{1}$ coincides with the previously introduced $\gamma_{H}$. The total force necessary to detach this type of tape/membrane can be computed as previously, by recursively adding the terms relative to the 
appropriate hierarchical level. For example, the second level of hierarchy can be described by adding to $\hat{\gamma}_{1}$ another term of the form

$$
\hat{\gamma}_{2}=\kappa_{2}\left(\cos \alpha_{2}-1+\sqrt{\left(1-\cos \alpha_{2}\right)^{2}+\lambda_{2} \hat{\gamma}}\right)^{2}
$$

where $\kappa_{2}, \lambda_{2}$ and $\alpha_{2}$ are analogous to the first level parameters $\kappa_{1}, \lambda_{1}$ and $\alpha_{1}$, respectively. Analogous expressions can be written for $i>2$. In order to compute the $\kappa_{i}$ and $\lambda_{i}$ and $\alpha_{i}$ parameters, it is necessary to consider the geometry (i.e. geometry and contact angles at the various hierarchical levels) of the new system. The approach outlined in the previous sections can then be adopted to determine higher order surface energy values $\gamma_{i}$ to the adhesive energy due to the additional hierarchical levels, and the corresponding peeling force. Given the small bending stiffness of the tapes at the various hierarchical levels, the angle variations from one hierarchical level to the next are in all cases small. Therefore, the corrections decrease in magnitude for an increasing number of levels, i.e. the adhesive energy and force values do not diverge. This can be seen in results illustrated in Fig. 6. Here, we consider as previously a perturbation $\epsilon$ on the contact angle from one level to the next, and assume for simplicity that the perturbation is of the same order for each level, i.e. $\cos \alpha_{i+1}=\cos \left(\alpha_{i}+\epsilon\right), \forall i$. Thus, an increase of the hierarchical level also implies an increase in the overall perturbation on the initial peeling angle $\alpha_{0}$. Figures $6 \mathrm{a}$ and $6 \mathrm{~b}$ show the effect of an increasing number of hierarchical levels for the $\hat{F}$ vs. $\alpha_{0}$ and $\hat{F}$ vs. $\hat{a}$ plots in the case of a hierarchical tape and a hierarchical axisymmetric membrane, réspectively. For 3 levels of hierarchy, at $\alpha_{0}=0.1$ the adhesive force is increased by approximately 6 times with respect to the non-hierarchical case. It is apparent that the main increase takes place for the first hierarchical levels, as is clearly visible in Figs. $6 \mathrm{c}$ and $6 \mathrm{~d}$, where $\hat{F}$ is plotted as a function of the number of hierarchical levels for fixed $\theta$ and $\hat{a}$ values, again in the case of a hierarchical tape and a hierarchical axisymmetric membrane, respectively. We can compute the gain in adhesive force at level $i$ by dividing $F_{i}$ by the force at level $i-1\left(F_{i-1}\right)$.

$$
\text { Gain }=\frac{F_{i}}{F_{i-1}}
$$

Plotting the gain values versus the hierarchical level for the simple tape and the axisymmetric membrane (Fig. 6 e, f), we see that after 2 or 3 levels, there is no further significant gain. Therefore, we can state that 2 or 3 hierarchical levels are sufficient to optimize adhesive force. A further increase in hierarchical levels could be detrimental, since the smallest features would become of the order of the characteristic size of the substrate roughness, leading to a decrease of adhesion 
209 [22]. This is consistent with observations on biological adhesive structures found in nature, such as 210 beetle legs or gecko toes [16][31], which typically display 2 or 3 levels of hierarchy. In the case of 211 octopus's sucker membranes, hairs appear to be present at most at three levels of hierarchy.

\section{Conclusions}

Understanding of the effect of a layer of hairs on the adhesive properties of octopus' suckers is important for the design of artificial suction cups with improved adhesion for various applications, such as smart-skin attachable skin patches [32] or biorobotic adhesive discs [33]. Here, we have evaluated the effect of hierarchical structure, i.e. the presence of hairs, on the adhesion and detachment of a simple tape and of an axisymmetric membrane, in order to gain insight into the adhesion mechanism of octopus' suckers (in particular the detachment of the acetabulum roof from the acetabulum wall). The model is based on a number of simplifying assumptions, e.g. that there is no hair bunching and that the peeling angle does not vary significantly between structures at one hierarchical level and those at the next. Furthermore, delamination is assumed to take place from a rigid substrate, whereas the real biological tissue considered is soft and relatively deformable. However, these assumptions are not expected to qualitatively modify the analysis herein.

Results for the simple tape case indicate that the presence of hairs can improve the adhesive properties by more than $30 \%$ at small peeling angles, with the effect decreasing for larger angles. This is consistent with observations on biological adhesion, where typically adhesive forces need to be enhanced at small peeling angles. The main parameter determining this increase is the initial detached length of the hairs, which has an upper limit in lengths for which there is an onset of bunching effects. The detachment force for an axisymmetric membrane also increases in the presence of hierarchical structuring. We show that the model can be easily extended to the analysis to multiple levels of hierarchy. Here, results indicate that the first hierarchical levels are the ones that contribute more to an increase in adhesive force. In terms of convergence, we find that after the third level of hierarchy there is no longer a significant change in peeling force.

This paper provides a possible explanation for the role of the hairs in octopus' suckers, correctly accounting for their role in determining the ON/OFF behavior during adhesion. Currently, further studies are under way to evaluate other possible functions of these hairs (e.g. sensing) that could be fundamental to the octopus functionality. Our work can also help the design of artificial suction 
239 cups by providing a model that predicts the potential benefits of a hierarchical surface in terms of 240 improved and angle-dependent adhesive properties.

\section{Acknowledgements}

\section{Author Contributions}

N.M.P designed the study and supervised the work, G.G. wrote the first draft of the manuscript and generated diagrams supervised also by F.B.. All the authors finalized the manuscript.

\section{Additional Information}

Competing Interests: The authors declare that they have no competing interests.

Data availability: The authors declare that the data supporting the findings of this study are available within the article and its supplementary information files. 
a)

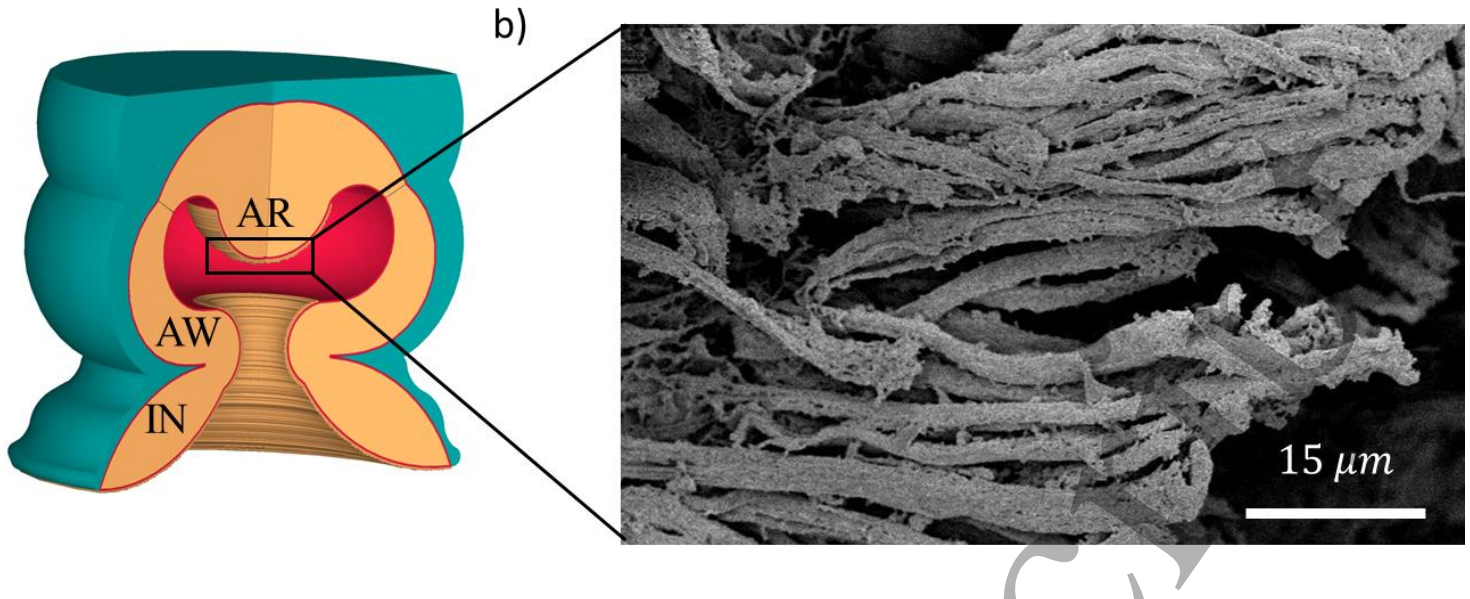

Figure 1: a) Schematic of the octopus' sucker: Acetabular Roof (AR), Acetabular Wall (AW) and Infundibulum (IN). b) Hairs present on the surface of the AR that is attached to the AW during adhesion.

19262

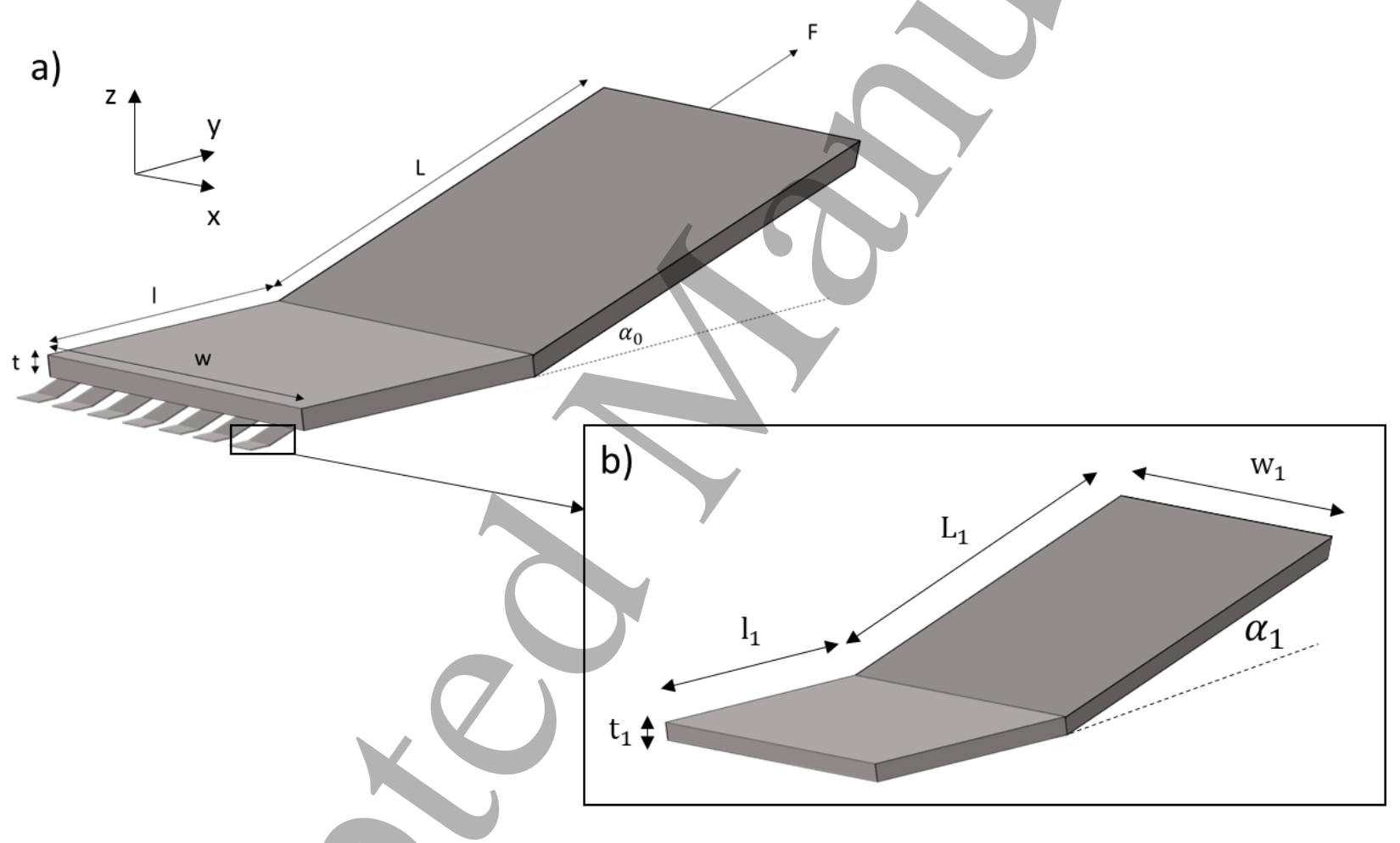

Figure 2: a) Schematic of the peeling of an elastic tape equipped with hairs; b) schematic of a single second -level tape (hair). 


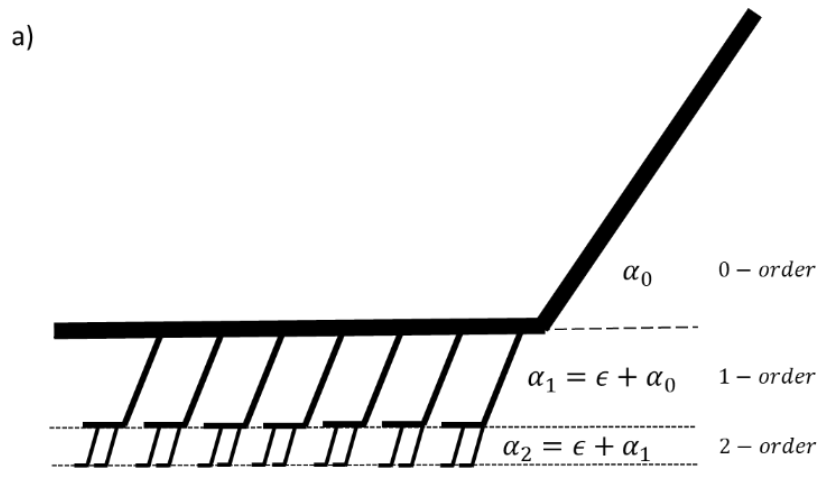

b)

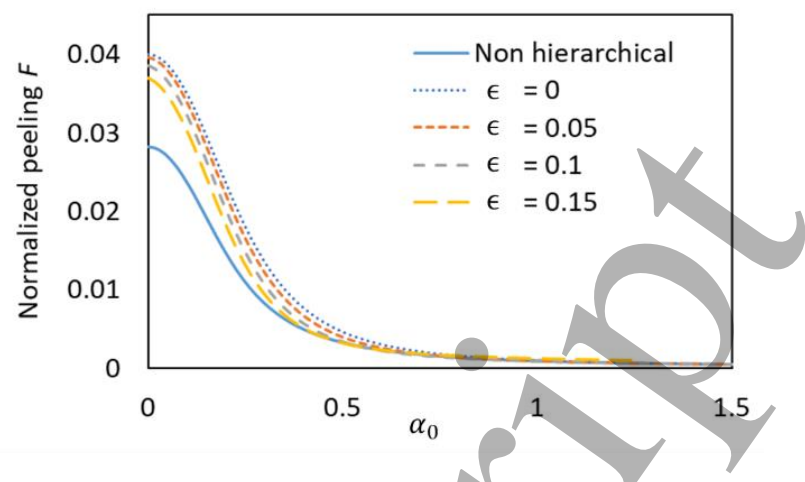

Figure 3: a) Schematic of hierarchical levels up to the second order. b) Normalized peeling force, vs. peeling angle (Eq. (7)) for different $\epsilon$ parameter values and $\hat{\gamma}=4 \cdot 10^{-4}$.

a)

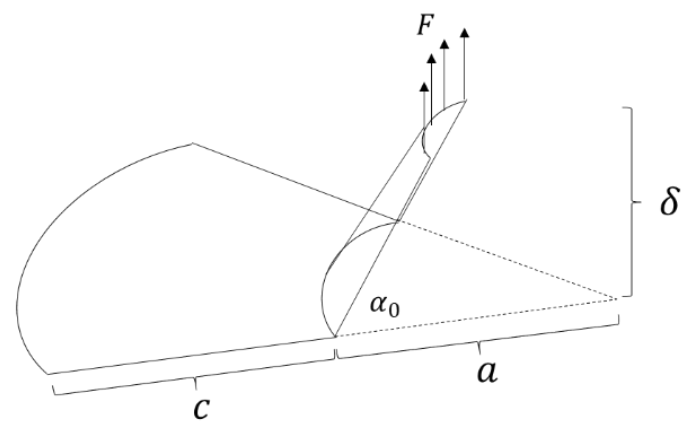

b)

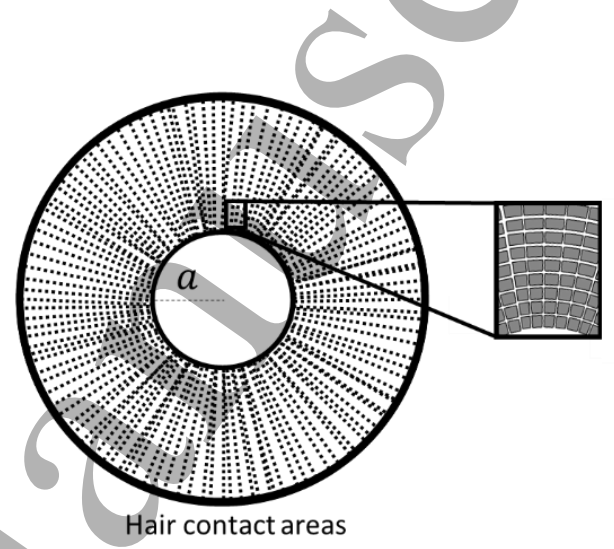

Figure 4: a) Schematic of the peeling of an axisymmetric membrane and b) schematic the contact region between the hairs of the axisymmetric membrane and the substrate. 


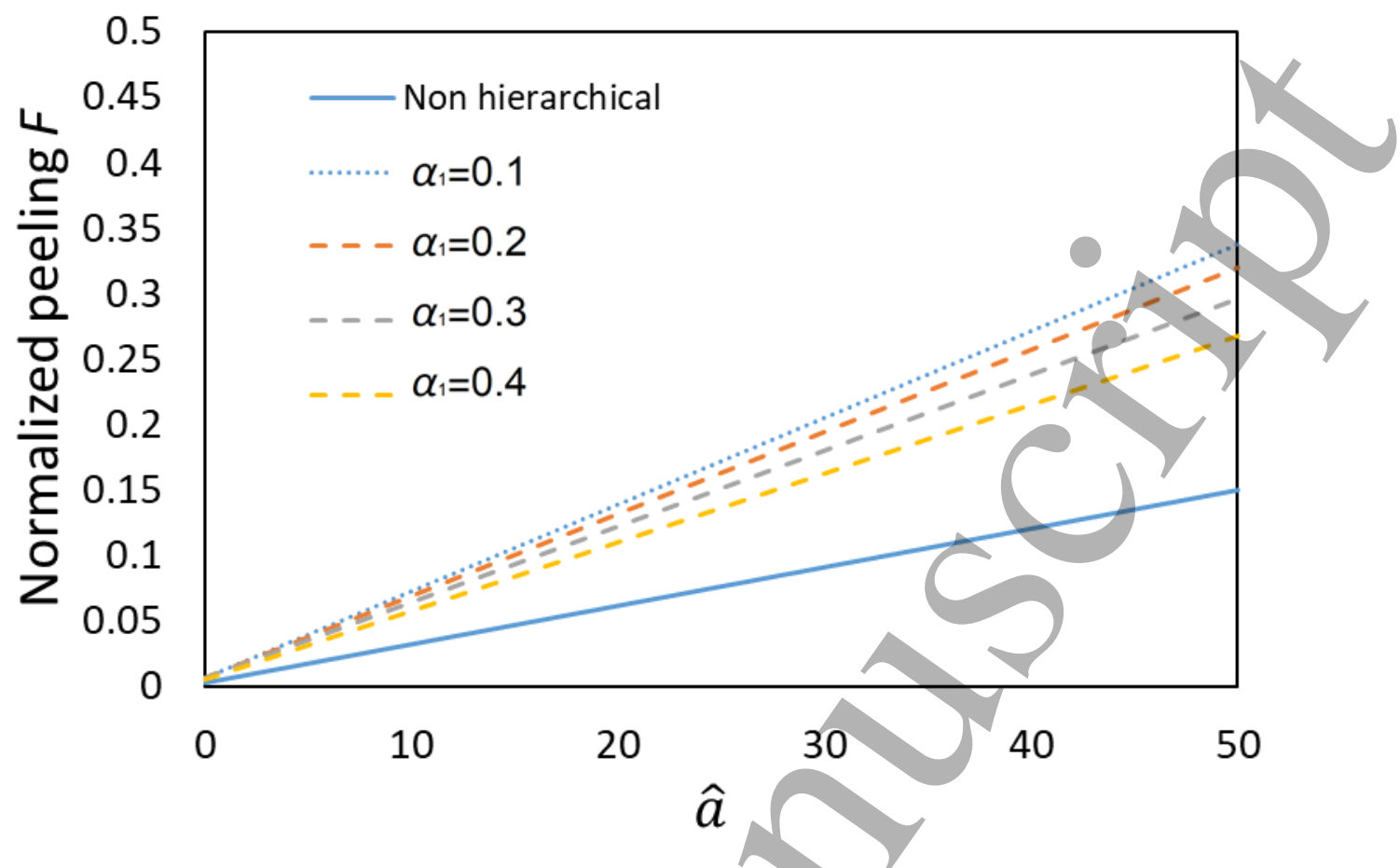

Figure 5: Peeling force for an axisymmetric membrane vs. detached radius $\hat{a}$ (Eq. (9)) for different $\alpha_{1}$ values $\left(\hat{\gamma}=4 \cdot 10^{-4}\right)$. 
a)

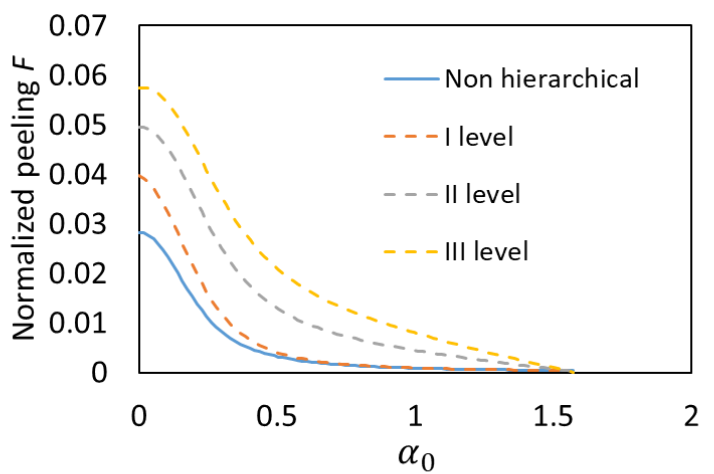

c)

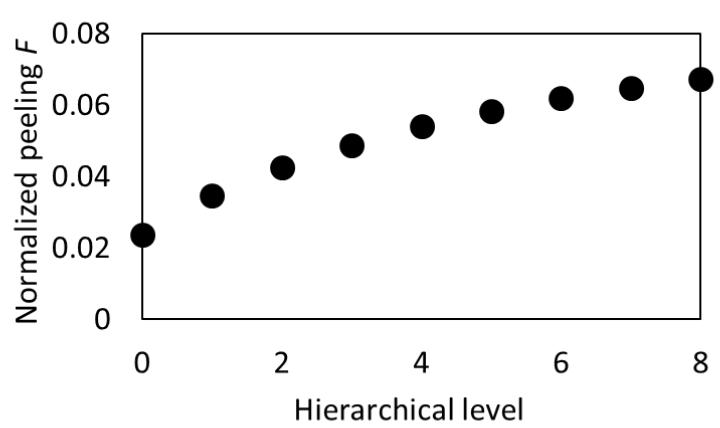

e)

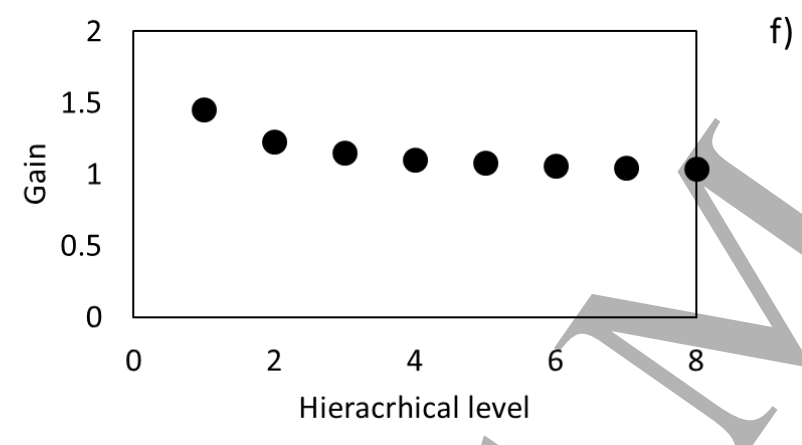

b)

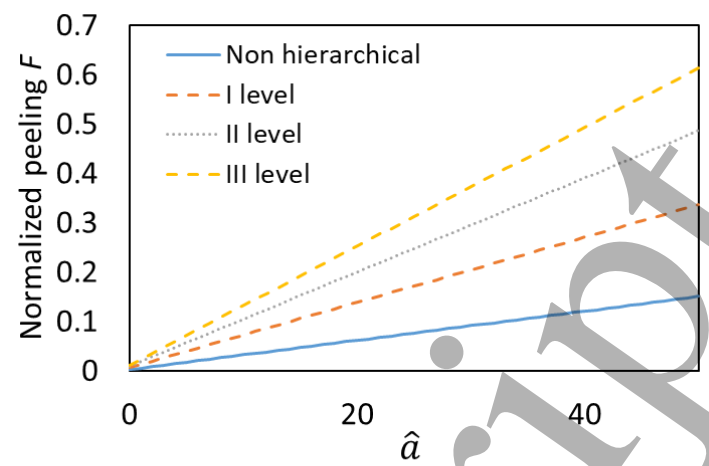

d)

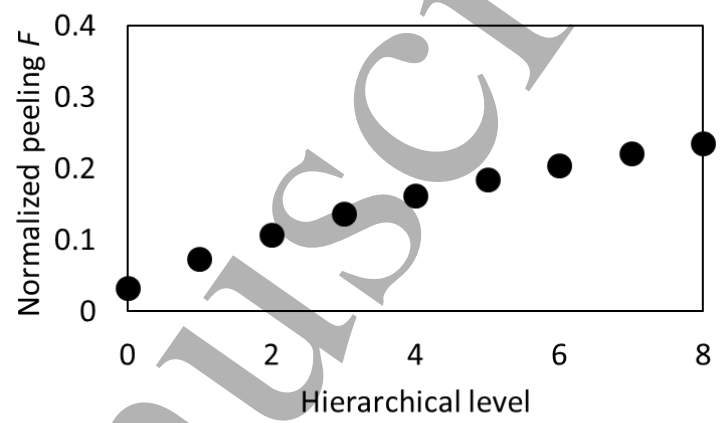

f)

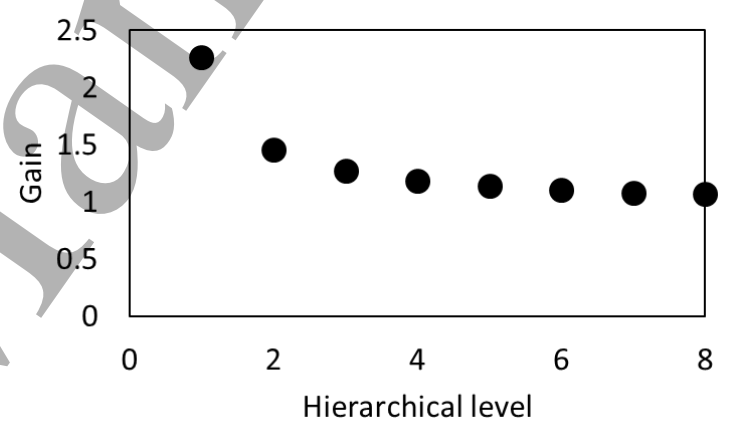

Figure 6: a) Normalized peeling force vs. peeling angle for increasing hierarchical levels in the case of a simple tape ( $\varepsilon=0.05)$; b) Normalized peeling force vs normalized detached radius for increasing hierarchical levels in the case of an axisymmetric membrane $\left(\varepsilon=0.05\right.$ and $\left.\alpha_{0}=0.1\right)$. c) Normalized peeling force as a function of number of hierarchical levels in the case of a simple tape ( $\varepsilon=0.05$ and $\alpha_{0}=0.1$ ). d) Normalized peeling force as a function of number of hierarchical levels in the case of an axisymmetric membrane $(\varepsilon=0.05, \hat{a}=10)$. e) Plot of the gain (Eq. 12) versus the hierarchical level for the simple tape and f) the axisymmetric membrane.

\section{References}

[1] L. A. Fuiman, C. M. Young, A. J. Gooday, and S. E. Shumway, Advances in Marine Biology. London, 2014.

[2] J. B. W. Roland C. Anderson, Jennifer A. Mather, Octopus The Ocean's Intelligente Invertebrate. Portland, 2010.

[3] A. M. Smith, "Cephalopod sucker design and the physical limits to negative pressure," J. Exp. Biol., vol. 199, no. Pt 4, pp. 949-58, 1996.

[4] J. A. Sandoval, S. Jadhav, H. Quan, D. D. Deheyn, and M. T. Tolley, "Reversible adhesion to rough surfaces both in and out of water, inspired by the clingfish suction disc," Bioinspir. 
Biomim., vol. 14, no. 6, 2019.

[5] F. Tramacere, M. Follador, N. M. Pugno, and B. Mazzolai, "Octopus-like suction cups: From natural to artificial solutions," Bioinspiration and Biomimetics, vol. 10, no. 3, pp. 1-8, 2015.

[6] M. Follador et al., "Octopus-inspired innovative suction cups," Lect. Notes Comput. Sci. (including Subser. Lect. Notes Artif. Intell. Lect. Notes Bioinformatics), vol. 8064 LNAI, pp. 368-370, 2013.

[7] S. Baik, D. W. Kim, Y. Park, T. J. Lee, S. Ho Bhang, and C. Pang, "A wet-tolerant adhesive patch inspired by protuberances in suction cups of octopi," Nature, vol. 546, no. 7658, pp. 396-400, 2017.

[8] S. Sareh et al., "Anchoring like octopus: biologically inspired soft artificial sucker," J. R. Soc. Interface, vol. 14, no. 135, p. 20170395, 2017.

[9] F. Tramacere, L. Beccai, M. J. Kuba, and B. Mazzolai, "Octopus Suckers Identification Code (OSIC)," Mar. Freshw. Behav. Physiol., vol. 46, no. 6, pp. 447-453, 2013.

[10] F. Tramacere, L. Beccai, M. Kuba, A. Gozzi, A. Bifone, and B. Mazzolai, "The Morphology and Adhesion Mechanism of Octopus vulgaris Suckers," PLoS One, vol. 8, no. 6, 2013.

[11] F. Tramacere, A. Kovalev, T. Kleinteich, S. N. Gorb, and B. Mazzolai, "Structure and mechanical properties of Octopus vulgaris suckers," J. R. Soc. Interface, vol. 11, no. 91, pp. 20130816-20130816, 2014.

[12] W. M. Kier, "The Structure and Adhesive Mechanism of Octopus Suckers," Integr. Comp. Biol., vol. 42, no. 6, pp. 1146-1153, 2002.

[13] F. Tramacere, N. M. Pugno, M. J. Kuba, B. Mazzolai, F. Tramacere, and B. Mazzolai, "Unveiling the morphology of the acetabulum in octopus suckers and its role in attachment," J. R. Soc. Interface, vol. 5, pp. 1-5, 2015.

[14] A. J. Kinloch, Adhesion and Adhesives. Heildeberg, 1987.

[15] K. Kendall, "Thin-Film Peeling - The Elastic Term," J. Phys. D. Appl. Phys., vol. 8, pp. 14491452, 1975.

[16] M. Varenberg, N. M. Pugno, and S. N. Gorb, "Spatulate structures in biological fibrillar adhesion," Soft Matter, vol. 6, no. 14, p. 3269, 2010.

[17] N. M. Pugno, "The theory of multiple peeling," Int. J. Fract., vol. 171, no. 2, pp. 185-193, 2011.

[18] J. O. Wolff, l. Grawe, M. Wirth, A. Karstedt, and S. N. Gorb, "Spider's super-glue: Thread anchors are composite adhesives with synergistic hierarchical organization," Soft Matter, 2015.

[19] J. O. Wolff and M. E. Herberstein, "Three-dimensional printing spiders: Back-and-forth glue application yields silk anchorages with high pull-off resistance under varying loading situations," J. R. Soc. Interface, 2017.

[20] L. Brely, F. Bosia, and N. M. Pugno, "Numerical implementation of multiple peeling theory and its application to spider web anchorages.," Interface Focus, vol. 5, no. 1, p. 20140051, 2015. 
[21] Z. L. Peng, S. H. Chen, and A. K. Soh, "Peeling behavior of a bio-inspired nano-film on a substrate," Int. J. Solids Struct., vol. 47, no. 14-15, pp. 1952-1960, 2010.

[22] L. Brely, F. Bosia, and N. M. Pugno, "The influence of substrate roughness, patterning, curvature, and compliance in peeling problems," Bioinspir. Biomim., vol. 13, no. 2, p. 026004, 2018.

[23] Z. Peng and S. Chen, "Effect of bending stiffness on the peeling behavior of an elastic thin film on a rigid substrate," Phys. Rev. E, vol. 042401, pp. 1-7, 2015.

[24] H. Gao, X. Wang, H. Yao, S. Gorb, and E. Arzt, "Mechanics of hierarchical adhesion structures of geckos," Mech. Mater., vol. 37, no. 2-3 SPEC. ISS., pp. 275-285, 2005.

[25] L. Brely, F. Bosia, and N. M. Pugno, "Emergence of the interplay between hierarchy and contact splitting in biological adhesion highlighted through a hierarchical shear lag model," Soft Matter, vol. 14, pp. 5509-5518, 2018.

[26] Z. Peng and S. Chen, "Effect of pre-tension on the peeling behavior of a bio-inspired nanofilm and a hierarchical adhesive structure," Appl. Phys. Lett., vol. 163702, no. September, 2012.

[27] F. Tramacere, E. Appel, B. Mazzolai, and S. N. Gorb, "Hairy suckers: The surface microstructure and its possible functional significance in the octopus vulgaris sucker," Beilstein J. Nanotechnol., vol. 5, no. 1, pp. 561-565, 2014.

[28] H. Yao and H. Gao, "Reprint of 'Multi-scale cohesive laws in hierarchical materials' [In. J. Solids Struct. 44 (2007) 8177-8193]," Int. J. Solids Struct., vol. 45, no. 13, pp. 3627-3643, 2008.

[29] B. Chen, P. . Wu, and H. Gao, "Hierarchical modelling of attachment and detachment mechanisms of gecko toe adhesion," Proc. R. Soc. A Math. Phys. Eng. Sci., vol. 464, no. 2094, pp. 1639-1652, 2008.

[30] L. Afferrante, G. Carbone, G. Demelio, and N. Pugno, "Adhesion of elastic thin films: Double peeling of tapes versus axisymmetric peeling of membranes," Tribol. Lett., vol. 52, no. 3, pp. 439-447, 2013.

[31] E. Arzt, S. Gorb, and R. Spolenak, "From micro to nano contacts in biological attachment devices," Proc. Natl. Acad. Sci. U. S. A., 2003.

[32] S. Baik, J. Kim, H. J. Lee, T. H. Lee, and C. Pang, "Highly Adaptable and Biocompatible Octopus-Like Adhesive Patches with Meniscus-Controlled Unfoldable 3D Microtips for Underwater Surface and Hairy Skin," Adv. Sci., 2018.

[33] Y. Wang et al., "A biorobotic adhesive disc for underwater hitchhiking inspired by the remora suckerfish," Sci. Robot., 2017. 